\title{
Depth Estimation Using the Tilt Angle of Gravity Field due to the Semi-Infinite Vertical Cylindrical Source
}

\begin{abstract}
Ata Eshaghzadeh
Institute of Geophysics, University of Tehran, Tehran, Iran

Correspondence should be addressed to Ata Eshaghzadeh; eshaghzadeh.ata@gmail.com

Received 24 July 2016; Revised 16 November 2016; Accepted 20 December 2016; Published 9 January 2017

Academic Editor: Umberta Tinivella

Copyright ( 2017 Ata Eshaghzadeh. This is an open access article distributed under the Creative Commons Attribution License, which permits unrestricted use, distribution, and reproduction in any medium, provided the original work is properly cited.

Tilt angle filter is an interpretation method that is used to determine the source borders locations from potential fields data. Moreover, the tilt angle is applied for estimation of the anomaly source depth, such as contact-depth method and tilt-depth method. In this paper an application of the tilt angle technique obtained from the first vertical and horizontal gradients of the gravity anomaly from semi-infinite vertical cylindrical source is described. The technique is based on the tilt angle and derivatives ratio. In this approach the depth estimates are proportional to the computed tilt angles and their distances from the cross section center of the anomaly cause on the surface. This new method is termed the tilt-distance-depth (TDD). The method is demonstrated using synthetic gravity data, with and without random noise, and real gravity data from Iran. The results are also compared with the solutions from Euler deconvolution technique and inverse modelling using Modelvision software.
\end{abstract}

\section{Introduction}

In most methods of potential fields modelling, the geological structures configuration is exhibited by simple geometrically shaped models. The models may not be geologically realistic, but usually approximate similarity to the gravity anomaly causative masses is sufficient for quantitative and qualitative interpretations and modelling. The geometric shapes of relevant some important geologic structures are similar to vertical cylinder such as igneous plugs, salt domes, and sink holes. The land and airborne gravimetry method are used worldwide for detection of these structures. Determination of the depth of the gravity anomaly sources is one of the main aims of quantitative interpretation of gravity field map. Many methods have been presented for calculating the depth. An excellent review is given in Nettleton [1]. Euler deconvolution gives initial estimates of source location and depth $[2,3]$. Abdelrahman and El-Araby [4], Abdelrahman et al. [5], and Essa [6] indicated that the window curves method can be used to estimate the depth and shape of the buried structure from gravity data. Abdelrahman et al. [7] and Abdelrahman and Abo-Ezz [8] developed a least-squares variance analysis method for shape and depth determination from gravity data.
The tilt angle has been used as the basis for a variety of methods for edge enhancement of potential field anomalies, such as Cooper [9, 10], Ferreira et al. [11], and Verduzco et al. [12]. Salem et al. [13] exhibited a contact-depth estimation approach based on the tilt angle of the magnetic data which was called the tilt-depth method. Cooper [14] proposed the contact-depth method based on the tilt angle for the automatic determination of the location, depth, and dip of contacts from aeromagnetic data. In this paper, an approach for the source depth determination based on the tilt angle of the $2 \mathrm{D}$ gravity data and its distance from origin, that is, the coordinate of the anomaly center on the profile, is presented. For shortness, this method is named the tilt-distance-depth (TDD) approach.

\section{Methodology}

Verduzco et al. [12] proposed the tilt angle filter. This filter is defined as

$$
T=\tan ^{-1}\left(\frac{\partial g / \partial z}{\sqrt{\left((\partial g / \partial x)^{2}+(\partial g / \partial y)^{2}\right)}}\right),
$$




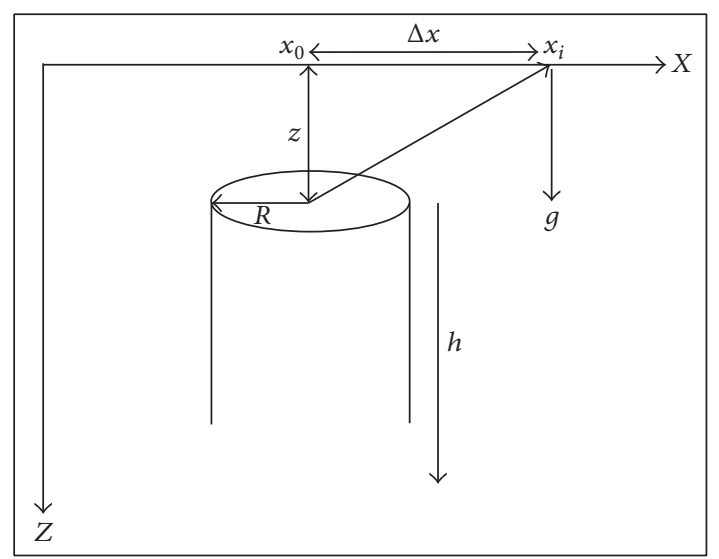

FIGURE 1: Semi-infinite vertical cylinder model.

where $T$ and $g$ are the tilt angle and gravity anomaly, respectively. The gravity anomaly expression produced by a semi-infinite vertical cylinder is given in Abdelrahman et al. $[15]$ as

$$
g=\frac{\pi G \rho R^{2}}{\left(x^{2}+z^{2}\right)^{1 / 2}}
$$

In (2), $z$ is the depth, $x$ is the position coordinate, $\rho$ is the density contrast, $G$ is the universal gravitational constant, and $R$ is the radius (Figure 1). For semi-infinite vertical cylinder model, depth to the top $(z)$ is much smaller than the source length ( $h$ ); that is, $z \ll h$ (Figure 1 ).

The first horizontal and vertical derivatives of $g$ are then

$$
\begin{aligned}
& \frac{\partial g}{\partial x}=\frac{-\pi G \rho R^{2} x}{\left(x^{2}+z^{2}\right)^{3 / 2}}, \\
& \frac{\partial g}{\partial z}=\frac{-\pi G \rho R^{2} z}{\left(x^{2}+z^{2}\right)^{3 / 2}} .
\end{aligned}
$$

Inserting (3) into (1), after simplification, yields

$$
T=\tan ^{-1}\left(\frac{z}{x}\right)
$$

therefore

$$
\frac{z}{x}=\tan T
$$

and consequently

$$
z=x \cdot \tan T
$$

Here, $x=\Delta x=x_{i}-x_{0}$ is the distance of the origin of the model from the gravity points (Figure 1). The coordinate of $x_{0}$ is correspondent to the coordinate of the maximum gravity value irregardless of its positive or negative sign. The $z$-axis is positive downward.

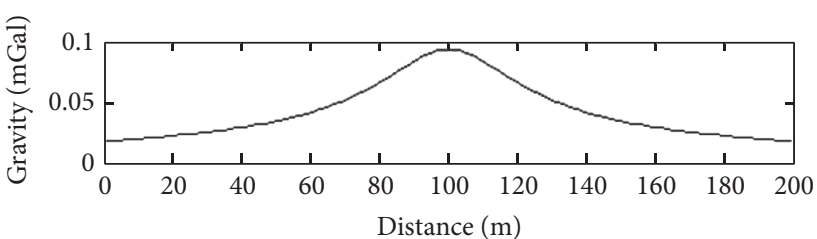

(a)

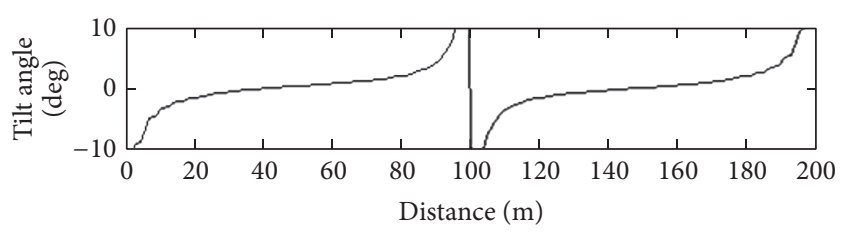

(b)

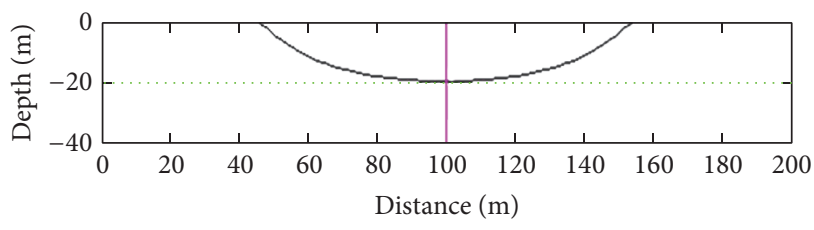

(c)

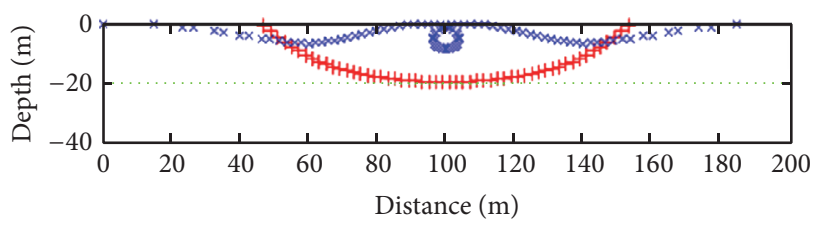

(d)

FIGURE 2: (a) Gravity anomaly from a semi-infinite vertical cylinder located at depth of $20 \mathrm{~m}$ and position of $100 \mathrm{~m}$. The model has radius of $40 \mathrm{~m}$ and density contrast $0.1 \mathrm{~g} / \mathrm{cm}^{3}$. (b) Tilt angle of data in (a). (c) Depth estimates from TDD method. The correct depth value $(20 \mathrm{~m})$ is drawn as a horizontal green dotted line. The vertical pink line shows the horizontal location of the source center. (d) Solutions from TDD and Euler deconvolution (using a structural index of 1 and a window size of 7 points) methods represented by red + and blue $\times$ symbols, respectively.

\section{Application to Synthetic Data}

Figure 2(a) shows the 2D synthetic gravity data set from a semi-infinite vertical cylinder located at depth of $20 \mathrm{~m}$ and position of $100 \mathrm{~m}$. The model has radius of $40 \mathrm{~m}$ and density contrast $0.1 \mathrm{~g} / \mathrm{cm}^{3}$. Figure 2(b) presents the tilt angle of the gravity data. The estimated depths are exhibited in Figure 2(c). The correct depth value $(20 \mathrm{~m})$ is drawn as a horizontal green dotted line. The vertical pink line shows the horizontal location of the source center. For comparison, solutions obtained from Euler deconvolution are also shown. In Figure 2(d) the solutions distribution obtained from TDD and Euler deconvolution methods are displayed as red + and blue $\times$ symbols, respectively. Solutions from Euler deconvolution have been obtained using a structural index of 1 and a window size of 7 points. The Euler method underestimates the depth to the top of the vertical cylinder. The sensitivity of 


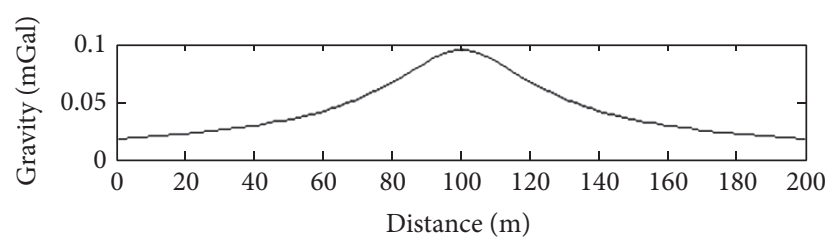

(a)

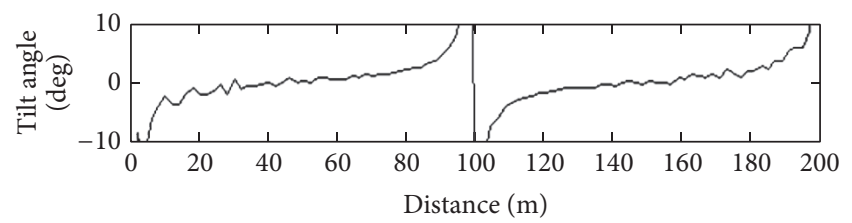

(b)

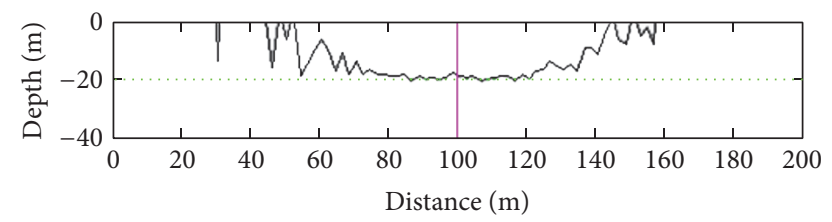

(c)

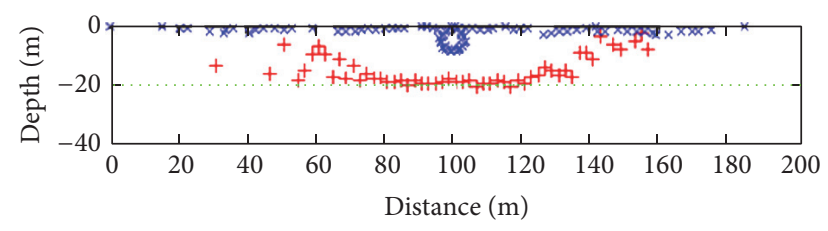

(d)

FIGURE 3: (a) Gravity anomaly of Figure 2(a) contaminated with random noise with standard deviation of $0.0001 \mathrm{mGal}$ and average $0.0005 \mathrm{mGal}$. (b) Tilt angle of data in the Figure 2(a). (c) Depth estimates from TDD method. The correct depth value $(20 \mathrm{~m})$ is drawn as a horizontal green dotted line. The vertical pink line shows the horizontal location of the source center. (d) Solutions from TDD and Euler deconvolution (using a structural index of 1 and a window size of 7 points) methods represented by red + and blue $\times$ symbols, respectively.

the TDD approach to noise is investigated in Figure 3. The gravity data in Figure 3(a) was contaminated with random noise with standard deviation of $0.0001 \mathrm{mGal}$ and average $0.0005 \mathrm{mGal}$ (Figure 3(a)). Figure 3(b) shows the tilt angle curve for gravity data in Figure 3(a). Figures 3(c) and 3(d) show the depth estimates using the TDD and Euler methods.

Figure 4(a) shows the synthetic gravity data profile from a semi-infinite vertical cylinder located at depth of $10 \mathrm{~m}$ and position of $100 \mathrm{~m}$. The model has radius of $30 \mathrm{~m}$ and density contrast $0.1 \mathrm{~g} / \mathrm{cm}^{3}$. The tilt angle of the gravity data in Figure 4(a) and the depth estimates are presented in Figures $4(\mathrm{~b}), 4(\mathrm{c})$, and 4(d), respectively. The correct depth value $(10 \mathrm{~m})$ is drawn as a horizontal green dotted line.

The vertical pink line shows the horizontal location of the source center. In Figure 4(d) solutions distribution from TDD and Euler deconvolution methods are displayed as red + and blue $\times$ symbols, respectively. For evaluating the performance of the TDD method a random noise with standard deviation of $0.0005 \mathrm{mGal}$ and average $0.001 \mathrm{mGal}$

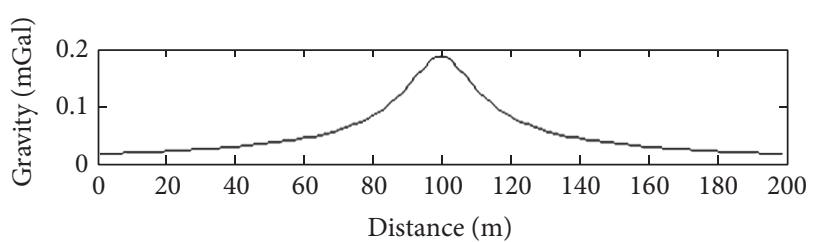

(a)

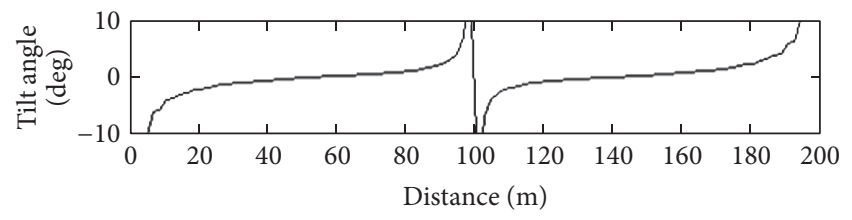

(b)

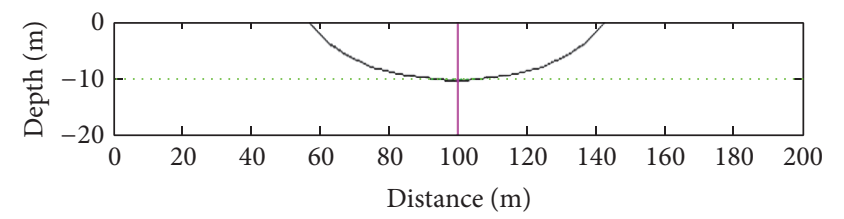

(c)

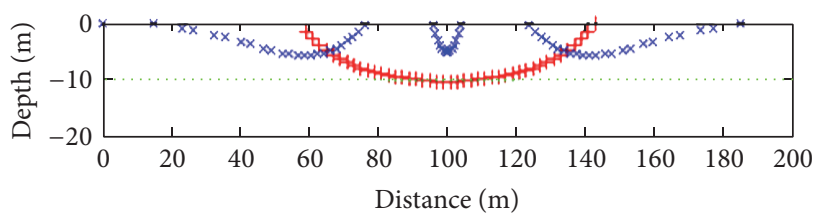

(d)

FIGURE 4: (a) Gravity anomaly from a semi-infinite vertical cylinder located at depth of $10 \mathrm{~m}$ and position of $100 \mathrm{~m}$. The model has radius of $30 \mathrm{~m}$ and density contrast $0.1 \mathrm{~g} / \mathrm{cm}^{3}$. (b) Tilt angle of data in (a). (c) Depth estimates from TDD method. The correct depth value $(20 \mathrm{~m})$ is drawn as a horizontal green dotted line. The vertical pink line shows the horizontal location of the source center. (d) Solutions from TDD and Euler deconvolution (using a structural index of 1 and a window size of 7 points) methods represented by red + and blue $\times$ symbols, respectively.

was added to the gravity data set (Figure 5(a)). Figures 5(b), $5(\mathrm{c})$, and 5(d) show the tilt angle of the gravity data in Figure 4(a) and the depth estimates.

The contiguous solutions with the same depth obtained from the TDD approach using the gravity data corrupted by random-valued noise indicate the exact depth value, as shown in Figures 3(d) and 5(d).

The depth estimates of the synthetic models, with and without random noise, for both methods have been given in Table 1 .

\section{Field Example}

The study region is located at the northwest of Iran. Miocene units in this region include sequences of marl, salt, and chalk. The salt domes in this area are the result of the upward movement of the Neogene evaporative materials. These salt domes are positioned at the shallow depth and have mostly high alloy. 


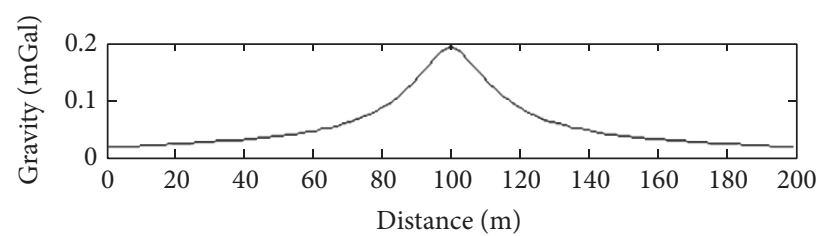

(a)

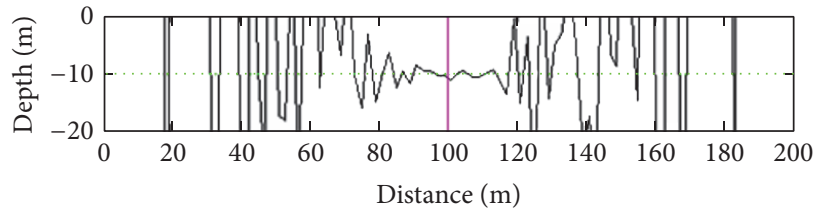

(c)

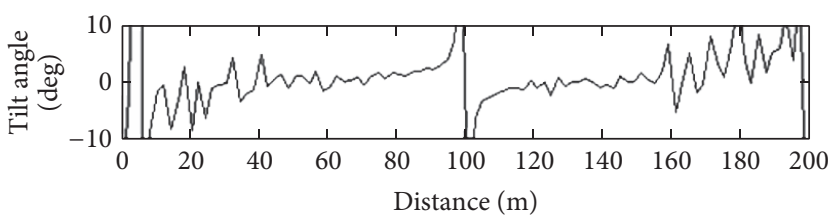

(b)

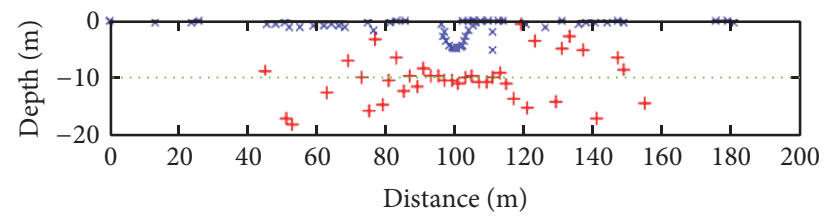

(d)

FIGURE 5: (a) Gravity anomaly of Figure 4(a) contaminated with random noise with standard deviation of $0.0005 \mathrm{mGal}$ and average $0.001 \mathrm{mGal}$. (b) Tilt angle of data in (a). (c) Depth estimates from TDD method. The correct depth value (20 m) is drawn as a horizontal green dotted line. The vertical pink line shows the horizontal location of the source center. (d) Solutions from TDD and Euler deconvolution (using a structural index of 1 and a window size of 7 points) methods represented by red + and blue $\times$ symbols, respectively.

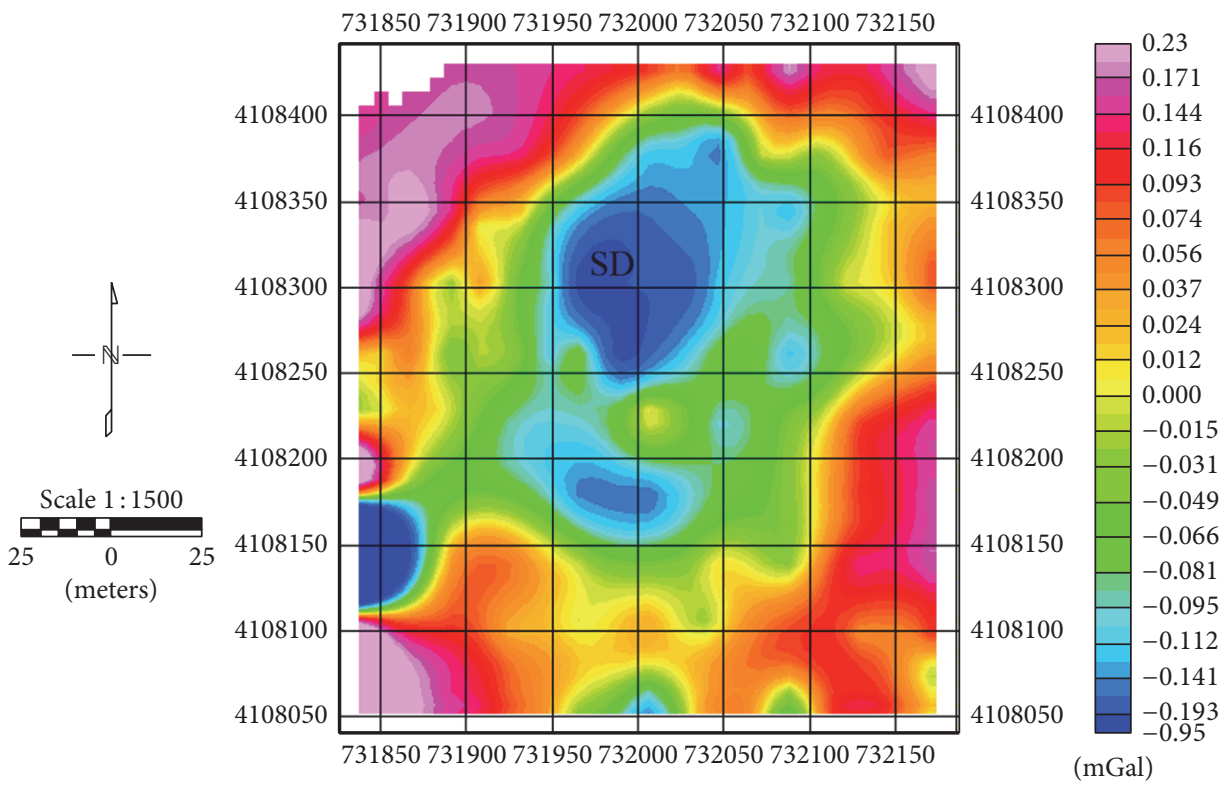

FIGURE 6: Residual gravity anomaly map. The region indicated by SD represents the salt dome location.

TABLE 1: The depth estimates of the synthetic models.

\begin{tabular}{lccc}
\hline \multirow{2}{*}{ Method } & \multirow{2}{*}{ Real depth $(\mathrm{m})$} & \multicolumn{2}{c}{ Estimated depth $(\mathrm{m})$} \\
& & Free noise & With noise \\
\hline TDD & 20 & 20 & 20 \\
TDD & 10 & 10 & 10 \\
Euler & 20 & 8 & 8 \\
Euler & 10 & 5 & 4 \\
\hline
\end{tabular}

Figure 6 shows the residual gravity anomaly from the exploratory region. The salt dome with negative anomaly is recognizable on the gravity map (SD on the Figure 6). For eliminating the short wavelengths, such as shallow and small anomaly sources and noise, the $10 \mathrm{~m}$ upward continuation filter was used (Figure 7). Figures 8(a) and 9(a) show the gravity field variations along profiles $A$ and $B$, respectively. Figures 8 (b) and 8(c) display the tilt angle of the gravity data and depth estimates from the TDD method along profile A and Figures 9(b) and 9(c) exhibit the tilt angle of the gravity data and depth estimates from the TDD method along profile B.

Solutions from the TDD and Euler deconvolution method are marked as red + and blue $\times$ symbols. The scattered form of the solutions has been exposed in Figures 8(d) and $9(\mathrm{~d})$.

The clustered and proximate solutions of the TDD procedure have computed the average depth of $25 \mathrm{~m}$ for the salt dome. It should be noted that the upward height has been subtracted from the depth estimates. 


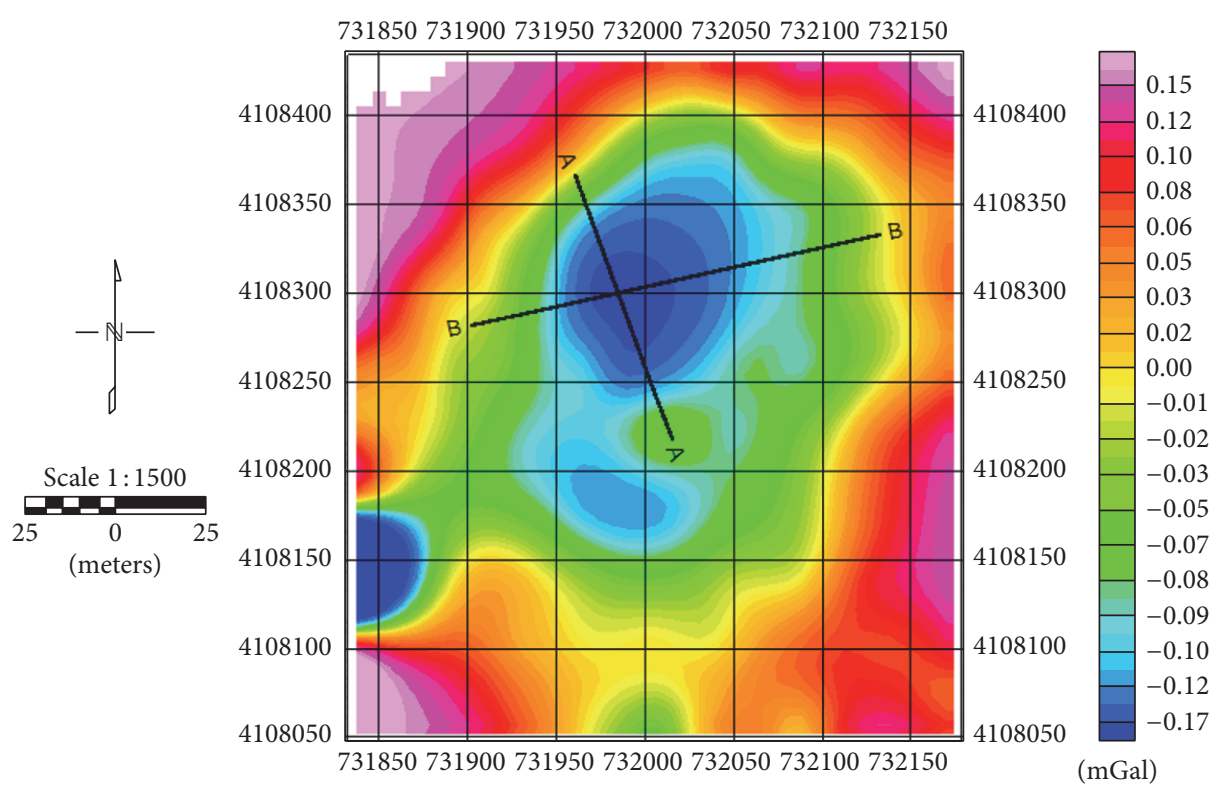

Figure 7: Upward continued to $10 \mathrm{~m}$ height of data in Figure 6. Two gravity profiles A and B cross over the salt dome structure.

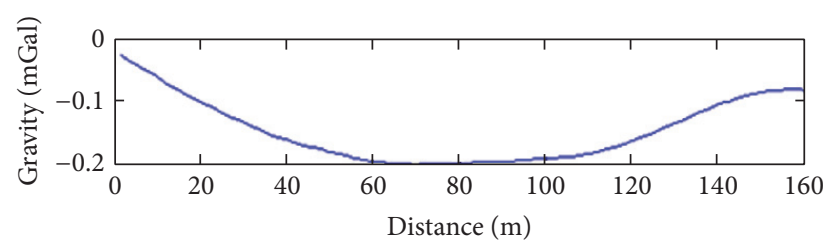

(a)

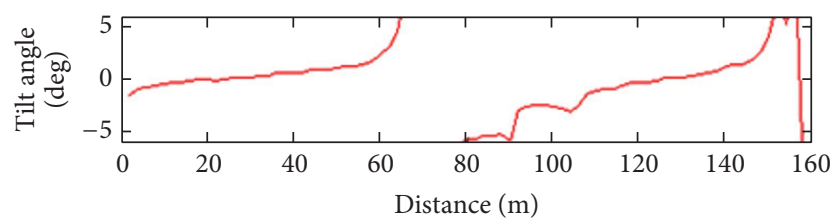

(b)

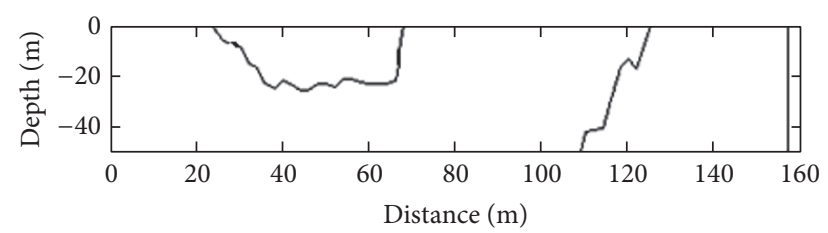

(c)

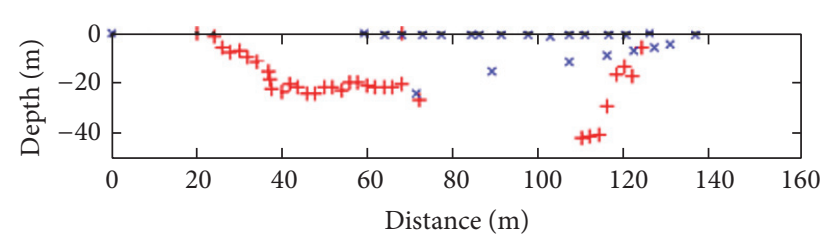

(d)

Figure 8: (a) Gravity profile A. The location of the profile is specified in Figure 7. (b) Tilt angle of gravity data in (a). (c) Depth estimates from TDD method. (d) Solutions from TDD and Euler deconvolution (using a structural index of 1 and a window size of $9 \times 9$ points) methods represented red + and blue $\times$ symbols, respectively.

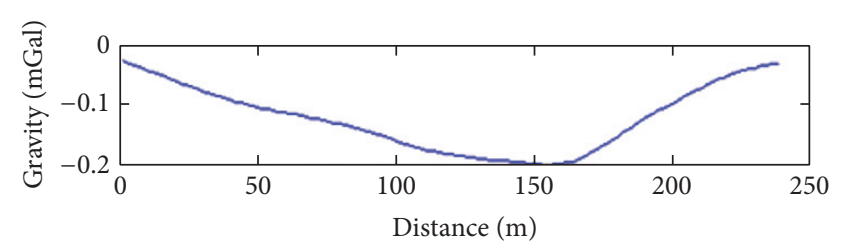

(a)

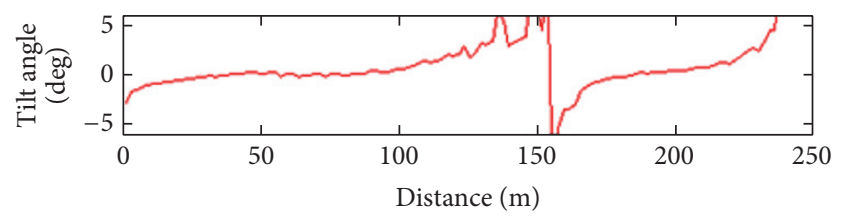

(b)

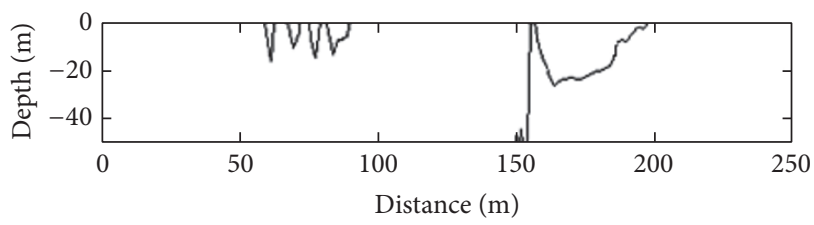

(c)

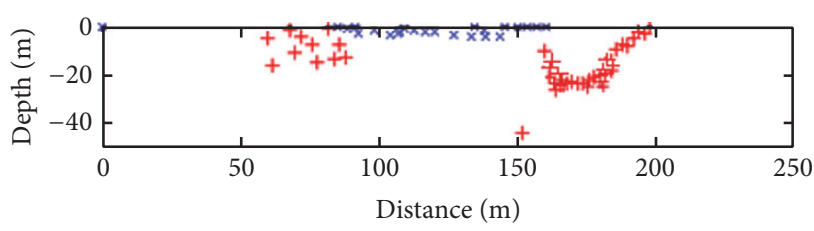

(d)

FIGURE 9: (a) Gravity profile A. The location of the profile has been specified in Figure 7. (b) Tilt angle of gravity data in (a). (c) Depth estimates from TDD method. (d) Solutions from TDD and Euler deconvolution (using a structural index of 1 and a window size of $9 \times 9$ points) methods represented red + and blue $\times$ symbols, respectively. 


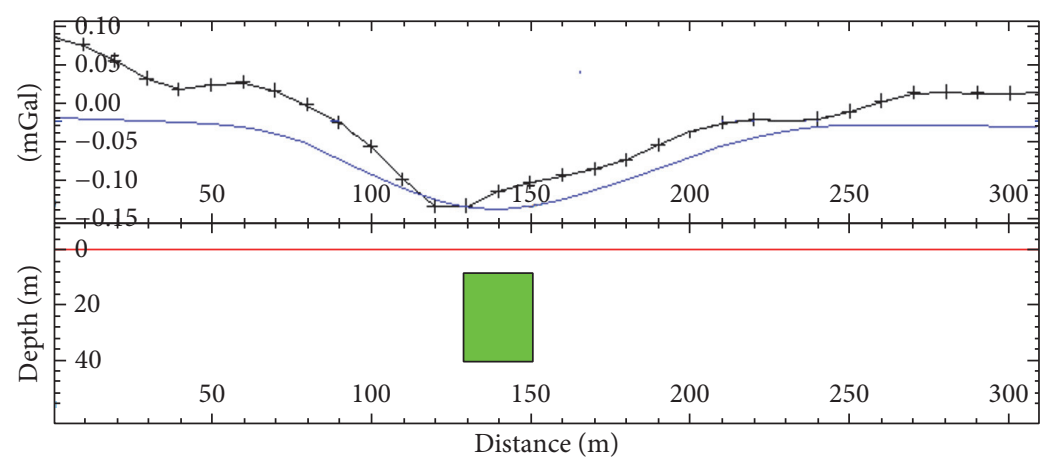

Figure 10: The initial model (profile of vertical cylinder) of a salt dome (green rectangle), gravity field changes through the profile 5 (black line), and calculated gravity of model (blue line) have been displayed.

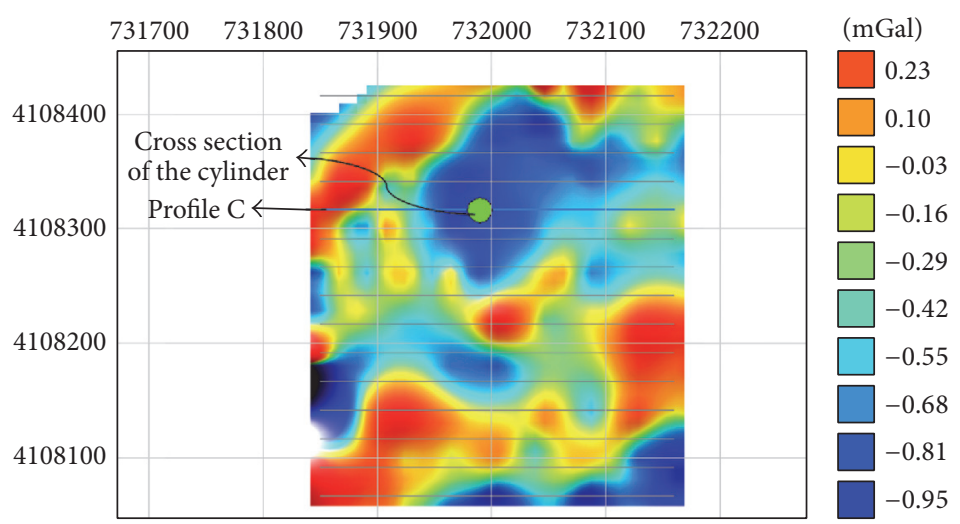

Figure 11: Map of residual gravity field. The cross section of the cylinder model is also shown (blue line). The inversion modelling has been done using gravity measured through profile C.

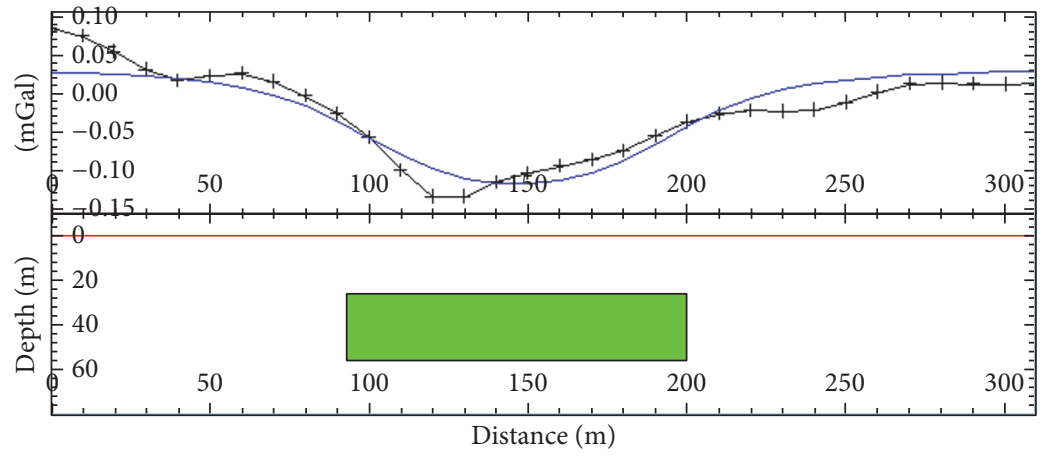

FIGURE 12: Vertical section of the final inversion model.

Moreover, the Modelvision software has been used for the inverse modelling of salt dome for estimating the upper depth of it. A profile of initial model (green rectangular) is depicted in Figure 10. As it can be seen that the graphs show the gravity field of the cylinder model (blue line) and variations of gravity caused by salt dome (black line), which has been measured through profile $\mathrm{C}$ shown in Figure 11. The location of cross section of initial model (green circle) has been presented in Figure 11.
In this model, the value of average density of the salt dome is assumed to be $2.2 \mathrm{gr} / \mathrm{cm}^{3}$. The final models are displayed through Figures 12 and 13. The estimated depths of upper and lower surface of cylinder model are $28 \mathrm{~m}$ and $58 \mathrm{~m}$, respectively. The estimations for the depth of the top of the salt dome by both approaches, that is, the proposed method TDD and inverse modelling, are almost equal.

Table 2 summarizes the average depth estimate results of the upper surface of the salt dome using the TDD and 


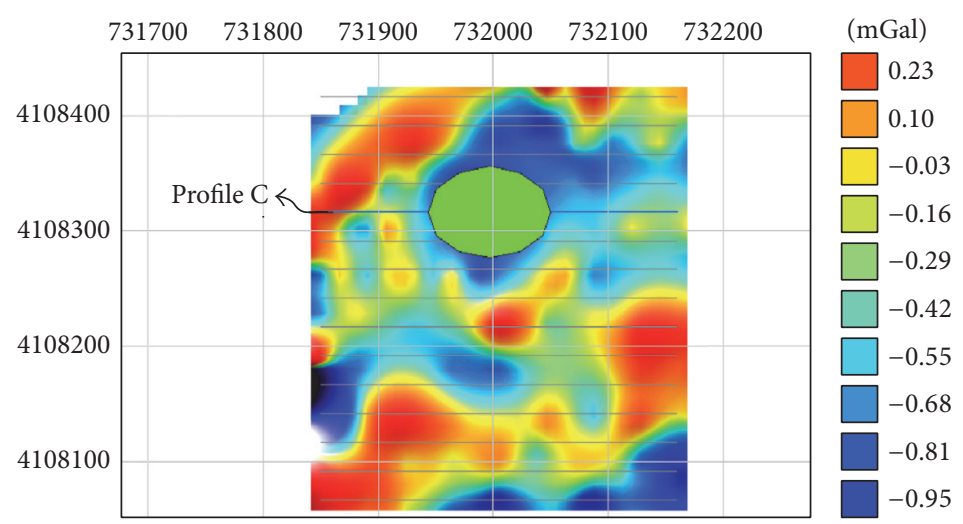

FIGURE 13: Horizontal section of the final inversion model using the gravity data of the profile C.

TABLE 2: The average depth estimates of the upper surface of the salt dome.

\begin{tabular}{lccc}
\hline Method & \multicolumn{3}{c}{$\begin{array}{c}\text { Estimated depth (m) } \\
\text { Profile B }\end{array}$} \\
\hline TDD & 24 & 25 & Profile C \\
Euler & $0-26$ & $0-6$ & - \\
Modelvision & - & - & 28 \\
\hline
\end{tabular}

Euler deconvolution methods and inverse modelling by the Modelvision software.

\section{Conclusions}

In this paper, a new depth estimation method based on the tilt angle of the gravity data has been presented. In comparison with the diffused solutions of the Euler deconvolution method which show a lower accuracy, the responses of the TDD method have high precision and with increasing the noise, the solutions remain stable. The results demonstrate the efficiency and usefulness of explained procedure. The results of the inverse modelling confirm the acceptable performance the TDD method.

\section{Competing Interests}

The author declares that they have no competing interests.

\section{References}

[1] L. L. Nettleton, Gravity and Magnetic in Oil Prospecting, McGraw-Hill Book Co, New York, NY, USA, 1976.

[2] D. T. Thompson, "EULDPH: a new technique for making computer-assisted depth estimates from magnetic data," Geophysics, vol. 47, no. 1, pp. 31-37, 1982.

[3] A. B. Reid, J. M. Allsop, H. Granser, A. J. Millett, and I. W. Somerton, "Magnetic interpretation in three dimensions using Euler deconvolution," Geophysics, vol. 55, no. 1, pp. 80-91, 1990.

[4] E. M. Abdelrahman and T. M. El-Araby, "Shape and depth solutions from moving average residual gravity anomalies," Journal of Applied Geophysics, vol. 36, no. 2-3, pp. 89-95, 1996.
[5] E. M. Abdelrahman, T. M. El-Araby, H. M. El-Araby, and E. R. Abo-Ezz, "A new method for shape and depth determinations from gravity data," Geophysics, vol. 66, no. 6, pp. 1774-1780, 2001.

[6] K. S. Essa, "Gravity data interpretation using the s-curves method," Journal of Geophysics and Engineering, vol. 4, no. 2, pp. 204-213, 2007.

[7] E. M. Abdelrahman, E. R. Abo-Ezz, K. S. Essa, T. M. El-Araby, and K. S. Soliman, "A least-squares variance analysis method for shape and depth estimation from gravity data," Journal of Geophysics and Engineering, vol. 3, no. 2, pp. 143-153, 2006.

[8] E. M. Abdelrahman and E. R. Abo-Ezz, "A least-squares standard deviation method to interpret gravity data due to finite vertical cylinders and sheets," Pure and Applied Geophysics, vol. 165, no. 5, pp. 947-965, 2008.

[9] G. R. J. Cooper and D. R. Cowan, "Enhancing potential field data using filters based on the local phase," Computers \& Geosciences, vol. 32, no. 10, pp. 1585-1591, 2006.

[10] G. R. J. Cooper, "Reply to a discussion about the 'Hyperbolic tilt angle method' by Zhou et al," Computers \& Geosciences, vol. 52, pp. 496-497, 2013.

[11] F. J. F. Ferreira, J. de Souza, A. D. B. E. S. Bongiolo, and L. G. de Castro, "Enhancement of the total horizontal gradient of magnetic anomalies using the tilt angle," GEOPHYSICS, vol. 78, no. 3, pp. J33-J41, 2013.

[12] B. Verduzco, J. D. Fairhead, C. M. Green, and C. MacKenzie, "New insights into magnetic derivatives for structural mapping," The Leading Edge, vol. 23, no. 2, pp. 116-119, 2004.

[13] A. Salem, S. Williams, J. D. Fairhead, D. Ravat, and R. Smith, "Tilt-depth method: a simple depth estimation method using first-order magnetic derivatives," The Leading Edge, vol. 26, no. 12, pp. 1502-1505, 2007.

[14] G. R. J. Cooper, "The automatic determination of the location, depth, and dip of contacts from aeromagnetic data," Geophysics, vol. 79, no. 3, pp. J35-J41, 2014.

[15] E. M. Abdelrahman, A. I. Bayoumi, Y. E. Abdelhady, M. M. Gobashy, and H. M. El-Araby, "Gravity interpretation using correlation factors between successive least-squares residual anomalies," Geophysics, vol. 54, no. 12, pp. 1614-1621, 1989. 

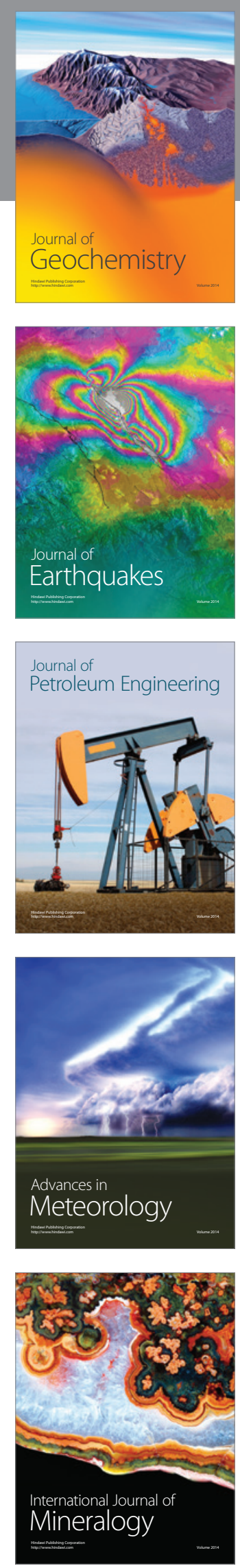
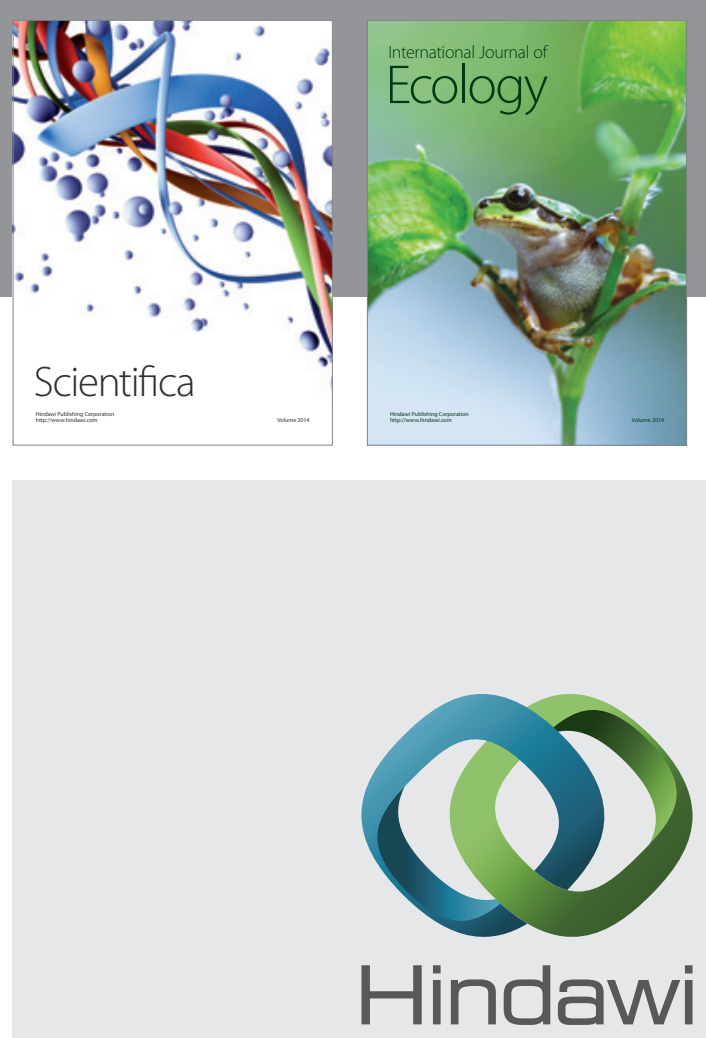

Submit your manuscripts at

https://www.hindawi.com
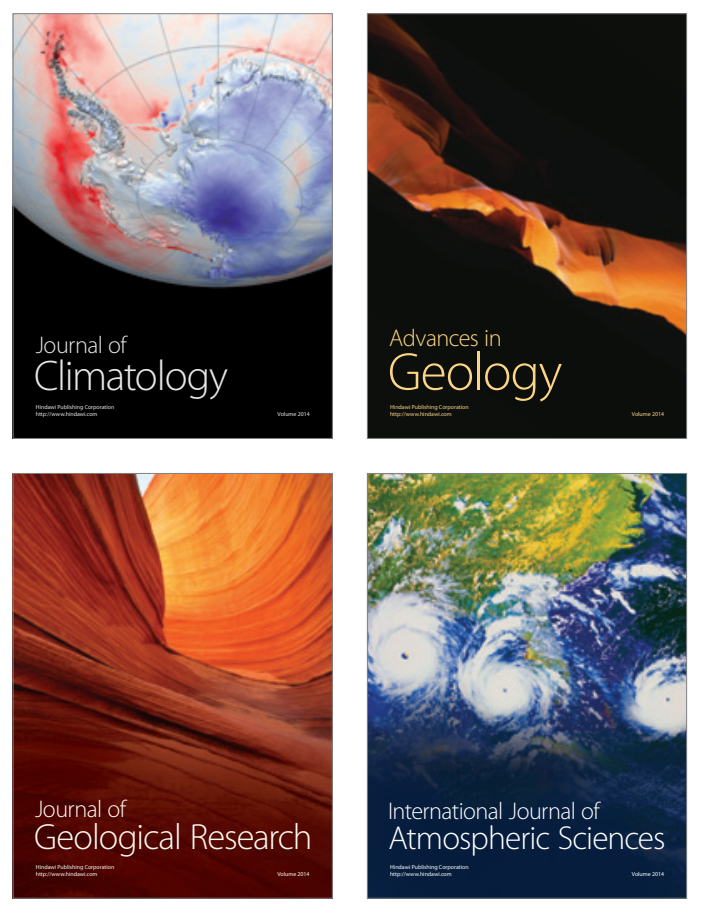

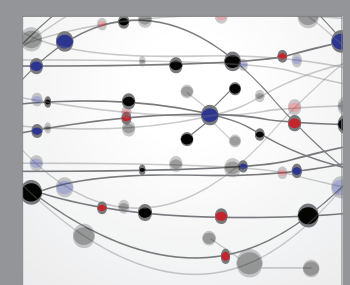

The Scientific

\section{World Journal}
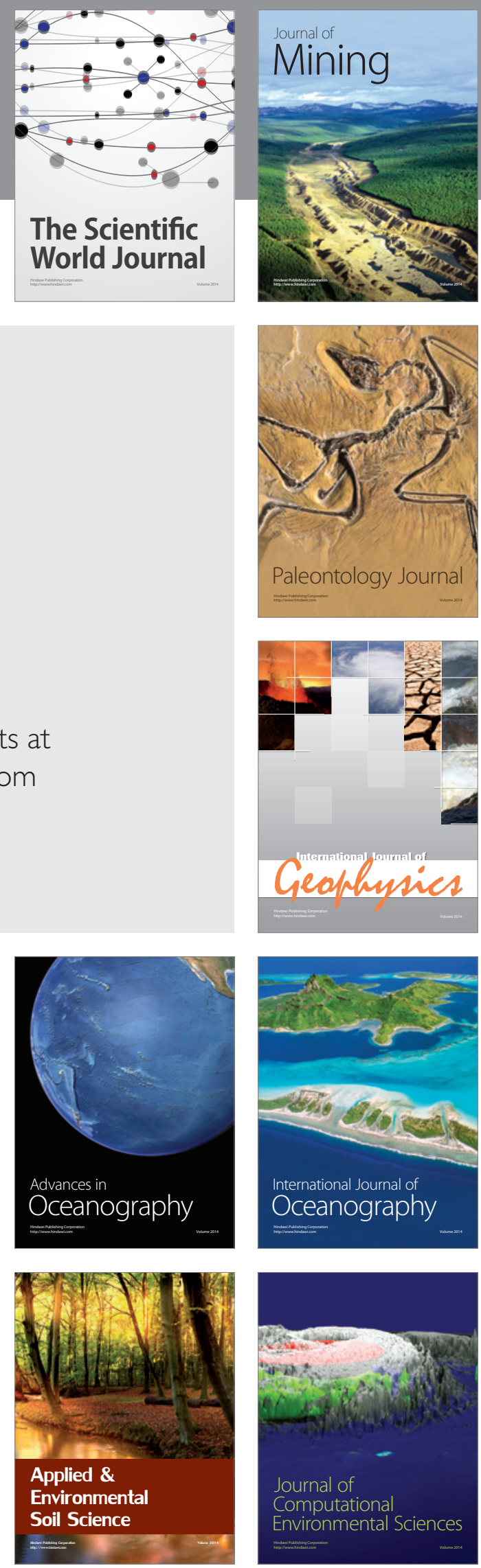